\title{
Janus kinase inhibitors and major COVID-19 outcomes: time to forget the two faces of Janus! A meta-analysis of randomized controlled trials
}

\author{
Dimitrios Patoulias $^{1}$ (1) $\cdot$ Michael Doumas $^{1,2} \cdot$ Christodoulos Papadopoulos $^{3} \cdot$ Asterios Karagiannis $^{1}$
}

Received: 1 August 2021 / Revised: 6 August 2021 / Accepted: 10 August 2021 / Published online: 24 August 2021

(C) International League of Associations for Rheumatology (ILAR) 2021

\begin{abstract}
Coronavirus disease-2019 (COVID-19) represents a global public health nightmare. The "cytokine storm," the most prominent underlying pathophysiologic mechanism of this disease, can theoretically be targeted at several stages. Janus kinase (JAK) inhibitors constitute a drug class that could ameliorate the inflammatory response and enhance antibody production. Herein, we aimed to evaluate the efficacy of JAK inhibitors in patients with COVID-19, performing the most updated relevant meta-analysis. We searched two major databases for randomized controlled trials (RCTs) enrolling adult patients with documented COVID-19 in the in-hospital setting, assigned either to JAK inhibitor treatment plus standard of care or standard of care alone. We set as primary efficacy outcome the endpoint of COVID-19 death on day 28 and as secondary efficacy composite outcome that of mechanical ventilation or initiation of extracorporeal membrane oxygenation (ECMO). We finally pooled data of interest from 4 RCTs in a total of 1338 subjects with documented COVID-19 infection, utilizing the following JAK inhibitors: baricitinib, ruxolitinib, tofacitinib, and nezulcitinib. Treatment with JAK inhibitor compared to control resulted in a significant reduction in the risk for COVID-19 death by $43 \%$, while it also led to a significant decrease in the risk for mechanical ventilation or ECMO initiation by $36 \%$. Herein, we demonstrate a clear benefit with JAK inhibitors added to standard of care in patients with COVID-19 in terms of risk reduction concerning major outcomes. Larger RCTs will elucidate their place in treatment armamentarium against COVID-19.
\end{abstract}

Key Points

- Coronavirus disease-2019 (COVID-19) remains a global health problem, currently unsolved since there is no established targeted treatment option.

- Janus kinase (JAK) inhibitors have been shown to ameliorate the so-called cytokine storm in the context of disease, while they might enhance antibody response.

- Herein, we present the most updated meta-analysis of randomized controlled trials, demonstrating that JAK inhibitors administered in the in-hospital setting produce a significant risk reduction concerning COVID-19 death by 43\%, compared to standard of care alone. In addition, their usage is associated with a significant decrease in the risk for mechanical ventilation or extracorporeal membrane oxygenation by $36 \%$.

- JAK inhibitors have provided promising results in COVID-19 therapeutic management so far. However, since evidence is considered as preliminary, further, larger clinical trials are warranted.

Keywords COVID-19 $\cdot$ Janus kinase inhibitor $\cdot$ Mechanical ventilation $\cdot$ Mortality

\section{Introduction}

COVID-19 remains a global nightmare, as it has affected almost 198 million people and has led to death nearly 4.2

Dimitrios Patoulias

dipatoulias@gmail.com

Extended author information available on the last page of the article million people worldwide, as of 1 August 2021. Despite the significant progress in diagnosis and treatment and the development of relevant vaccines, COVID-19 pandemic still poses a major burden for National Health Systems across the world. Corticosteroids represent the only drug class that has been shown to improve surrogate outcomes during disease course, namely mechanical ventilation and death [1]. Cytokine storm has now been recognized as the major pathophysiologic process for the development of 
main disease complications, unraveling a number of different treatment targets at various stages of its progression $[2,3]$.

Janus kinase (JAK) inhibitors constitute such a treatment option, which has provided a significant improvement in surrogate COVID-19 outcomes in relevant observational studies, while some of the most recent meta-analyses of them have proven their efficacy $[4,5]$. A significant reduction in circulating levels of inflammatory cytokines, a rapid recovery of circulating $\mathrm{T}$ - and B-cell frequencies, and increased antibody production against the SARS-CoV-2 spike protein have been proven with baricitinib use, a JAK inhibitor, in patients with severe COVID-19, as demonstrated in two observational studies from Italy $[6,7]$.

Therefore, since randomized controlled trials (RCTs) remain the mainstay of the evaluation of safety and efficacy of various treatment options, we sought to determine whether JAK inhibitors may provide improvement in COVID-19 major outcomes of interest.

\section{Methods}

We searched PubMed and Cochrane Library databases for relevant published RCTs up to 30 July 2021. We searched for randomized controlled trials enrolling adult hospitalized patients with documented SARS-CoV-2 infection, comparing the safety and efficacy of JAK inhibitors added to standard of care compared to standard of care alone. We excluded observational studies (retrospective or prospective) and studies enrolling pediatric population, if any. We did not set any exclusion criterion according to sample size of eligible randomized controlled trials or route of JAK inhibitor administration. We did not impose any filter regarding study language or text availability.

We set as primary efficacy outcome the endpoint of COVID-19 death on day 28 and as secondary efficacy composite outcome that of mechanical ventilation or initiation of extracorporeal membrane oxygenation (ECMO).

We implemented the following search strategy: $(((()(($ Janus Kinase Inhibitor) OR (JAK inhibitor)) OR (tofacitinib)) OR (ruxolitinib)) OR (baricitinib)) OR (oclacitinib)) OR (peficitinib)) OR (filgotinib)) OR (fedratinib) OR (nezulcitinib) AND (((COVID-19) OR (COVID19)) OR (coronavirus disease)) OR (SARS-CoV-2). Two independent reviewers (D.P. and C.P.) extracted the data from the eligible reports, by using a pilot tested, data extraction form.

As we assessed only dichotomous variables, differences were calculated with the use of risk ratio (RR), with $95 \%$ confidence interval (CI), after implementation of the MantelHaenszel $(\mathrm{M}-\mathrm{H})$ random effects formula. Statistical heterogeneity among studies was assessed by using $I^{2}$ statistics. All analyses were performed at the 0.05 significance level, while they were undertaken with RevMan 5.3 software.

Two independent reviewers (D.P. and M.D.) assessed the quality of the included RCTs, by using the Revised Cochrane risk of bias tool for randomized trials (RoB 2.0) for the primary efficacy outcome [8]. Discrepancies between reviewers were solved by discussion, consensus, or arbitration by a third senior reviewer (A.K.).

Finally, grading of retrieved evidence for the primary efficacy outcome was performed according to GRADE framework [9].

\section{Results}

We initially retrieved 346 results from PubMed database and 56 results from Cochrane Library database.

After de-duplication and screening at title and abstract level, we finally pooled data of interest from 4 RCTs [10-13] in at total of 1338 subjects with documented COVID-19 infection, utilizing the following JAK inhibitors: baricitinib and ruxolitinib, both being JAK $1 / 2$ inhibitors, and tofacitinib, which represents a JAK $1 / 3$ inhibitor with potential selectivity against JAK 2 , and nezulcitinib, designed to target all JAK isoforms. Baricitinib and tofacitinib were administered intravenously, ruxolitinib was given orally, while nezulcitinib was given via inhalation. Study selection process is depicted in detail in the corresponding flow diagram in supplementary figure 1.

Participants' baseline characteristics of interest are summarized in supplementary table 1 . One study was conducted in Brazil, one study was conducted in China, while the remaining two enrolled patients from different countries (Moldova, Ukraine, UK, Singapore, South Korea, Mexico, Japan, Spain, Denmark, USA). As shown in supplementary table 1, all RCTs were performed in the in-hospital setting, enrolling patients predominantly male (almost two-third of all enrolled subjects), aged from 54 to 62 years old, overweight, or obese, according to their body mass index. A significant proportion of subjects suffered from concomitant hypertension (ranging from 39 to 68\%) and diabetes mellitus (ranging from 19 to $38 \%$ ), while documentation of the rest major comorbidities was inadequate. Usage rates of glucocorticoids in the context of standard of care ranged from 70 to $100 \%$, while a significant proportion of enrolled participants received antiviral treatment, mainly remdesivir or oseltamivir.

As shown in Fig. 1, treatment with JAK inhibitor compared to control resulted in a significant reduction in the risk for COVID-19 death by $43 \%$ (RR $=0.57,95 \%$ CI; $0.37-0.89$, $I^{2}=0 \%$ ). In addition, JAK inhibitors compared to control led to a significant decrease in the risk for mechanical ventilation 


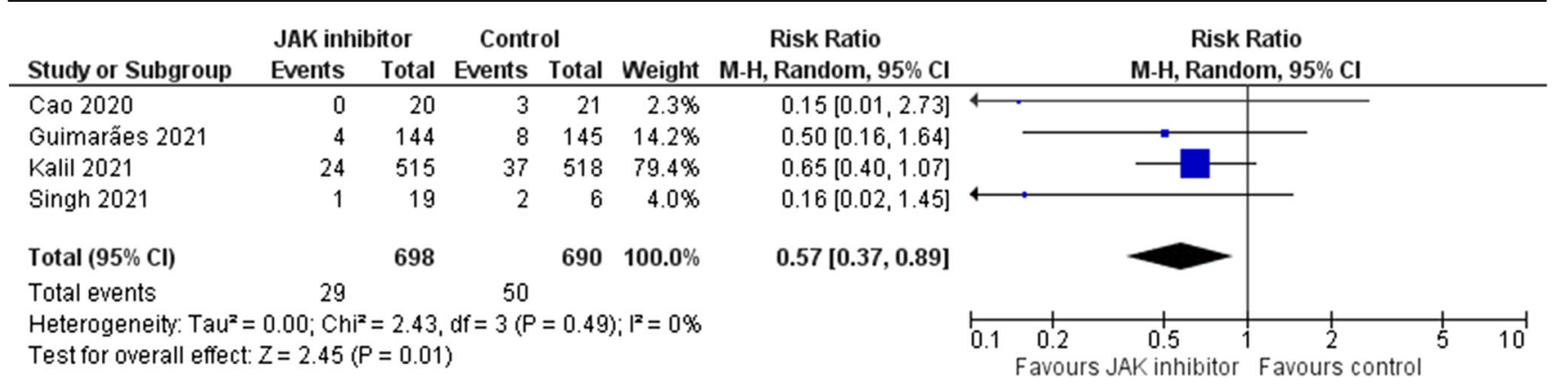

Fig. 1 Effect of JAK inhibitors compared to control on the risk for COVID-19 death

or ECMO initiation by $36 \%(\mathrm{RR}=0.64,95 \% \mathrm{CI}$; $0.47-0.89$, $I^{2}=0 \%$ ), as depicted in Fig. 2. No heterogeneity was demonstrated for any of the two aforementioned comparisons.

Risk of bias assessment, provided in supplementary table 2 , is overall considered as low across the selected studies, despite the fact that the randomization process risk of bias was evaluated as unclear for two RCTs $[11,13]$. Evidence concerning the primary efficacy outcome, namely COVID-19 death, was graded as moderate (supplementary summary of findings table).

Limitations We consider as major limitations of our metaanalysis the small number of the included trials and the lack of adequate reporting to perform additional analyses, according to baseline characteristics of interest (age, gender, comorbidities). In addition, we did not prospectively register a systematic review and meta-analysis protocol in a publicly available repository.

\section{Conclusion}

This brief meta-analysis of 4 RCTs investigating the effect of JAK inhibitors on the risk for COVID-19 death and mechanical ventilation/initiation of ECMO demonstrated a clear benefit with this drug class, when added to standard of care. These results are both interesting and promising, as they might encourage the usage of JAK inhibitors in patients hospitalized with severe disease. Of course, these results should be interpreted with caution, since the number of studies and enrolled subjects are small. In addition, it would be of interest to assess their clinical efficacy regarding major outcomes in patients infected with the newly identified SARS-CoV-2 variants, such as delta variant, which now account for the vast majority of newly diagnosed cases worldwide. Of course, their efficacy might also be tested in the pediatric population, since there are only some sparse relevant reports in the literature [14]. Another question that arises is whether this drug class has a clear and direct impact on associated COVID-19 complications, such as coagulopathy in the context of the disease [15]. Finally, it would be interesting and crucial, in terms of clinical decision-making, whether JAK inhibitors provide greater efficacy against severe disease and major complications, compared to other immunomodulatory agents, such as anti-interleukins [16].

Further, larger RCTs are required to confirm these important and noteworthy results in the long run of the therapeutic management of infected patients in the context of COVID-19 pandemic.

Supplementary Information The online version contains supplementary material available at https://doi.org/10.1007/s10067-021-05884-4.

Author contribution Dimitrios Patoulias and Michael Doumas conceived and designed the study. Dimitrios Patoulias, Michael Doumas, and Christodoulos Papadopoulos organized search strategy, performed literature screening, extracted data of interest, and assessed risk of bias. Dimitrios Patoulias did the analyses. Dimitrios Patoulias and Michael Doumas wrote the first draft. Asterios Karagiannis critically revised the manuscript. All authors agree to its submission.

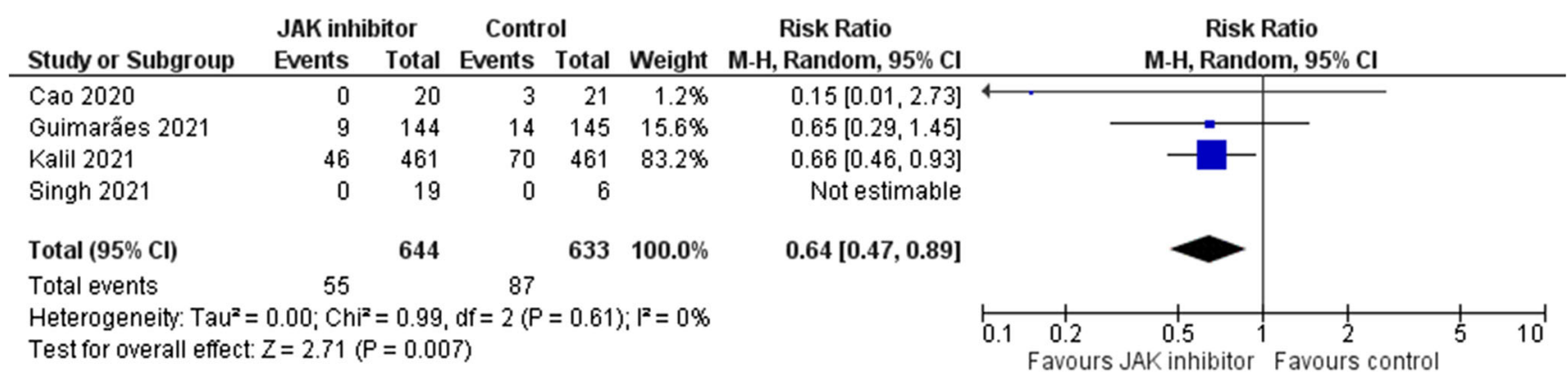

Fig. 2 Effect of JAK inhibitors compared to control on the risk for mechanical ventilation or ECMO initiation in the context of COVID-19 


\section{Compliance with ethical standards}

Disclosures None.

\section{References}

1. Siemieniuk RA, Bartoszko JJ, Ge L, Zeraatkar D, Izcovich A, Kum E, Pardo-Hernandez H, Rochwerg B, Lamontagne F, Han MA, Liu Q, Agarwal A, Agoritsas T, Chu DK, Couban R, Darzi A, Devji T, Fang B, Fang C et al (2020) Drug treatments for COVID-19: living systematic review and network meta-analysis. Update in. BMJ 371: $\mathrm{m} 4852$

2. Fajgenbaum DC, June CH (2020) Cytokine storm. N Engl J Med 383:2255-2273. https://doi.org/10.1056/NEJMra2026131

3. Morris G, Bortolasci CC, Puri BK, Marx W, O’Neil A, Athan E, Walder K, Berk M, Olive L, Carvalho AF, Maes M (2021) The cytokine storms of COVID-19, H1N1 influenza, CRS and MAS compared. Can one sized treatment fit all? Cytokine 144:155593. https://doi.org/10.1016/j.cyto.2021.155593

4. Chen CX, Wang JJ, Li H, Yuan LT, Gale RP, Liang Y (2021) JAKinhibitors for coronavirus disease-2019 (COVID-19): a meta-analysis [published online ahead of print, 2021 May 14]. Leukemia 2021:1-5. https://doi.org/10.1038/s41375-021-01266-6.

5. Wijaya I, Andhika R, Huang I, Purwiga A, Budiman KY, Bashari MH, Reniarti L, Roesli RMA (2021) The use of Janus kinase inhibitors in hospitalized patients with COVID-19: systematic review and meta-analysis. Clin Epidemiol Glob Health 11:100755. https:// doi.org/10.1016/j.cegh.2021.100755

6. Bronte V, Ugel S, Tinazzi E, Vella A, De Sanctis F, Canè S, Batani V, Trovato R, Fiore A, Petrova V, Hofer F, Barouni RM, Musiu C, Caligola S, Pinton L, Torroni L, Polati E, Donadello K, Friso S et al (2020) Baricitinib restrains the immune dysregulation in patients with severe COVID-19. J Clin Invest 130:6409-6416. https://doi. org $/ 10.1172 / \mathrm{JCI} 141772$

7. Vannucchi AM, Sordi B, Morettini A, Nozzoli C, Poggesi L, Pieralli F, Bartoloni A, Atanasio A, Miselli F, Paoli C, Loscocco GG, Fanelli A, Para O, Berni A, Tassinari I, Zammarchi L, Maggi L, Mazzoni A, Scotti V et al (2021) Compassionate use of JAK1/2 inhibitor ruxolitinib for severe COVID-19: a prospective observational study. Leukemia 35:1121-1133. https://doi.org/10.1038/ s41375-020-01018-y

8. Higgins JPT, Sterne JAC, Savović J, Page MJ, Hróbjartsson A, Boutron I, Reeves B, Eldridge S (2016) A revised tool for assessing risk of bias in randomized trials. Cochrane Database Syst Rev 10: 29-31

9. Brozek JL, Akl EA, Alonso-Coello P, Lang D, Jaeschke R, Williams JW, Phillips B, Lelgemann M, Lethaby A, Bousquet J,
Guyatt GH, Schünemann HJ, GRADE Working Group (2009) Grading quality of evidence and strength of recommendations in clinical practice guidelines. Part 1 of 3 . An overview of the GRADE approach and grading quality of evidence about interventions. Allergy 64:669-677. https://doi.org/10.1111/j.1398-9995. 2009.01973.x

10. Kalil AC, Patterson TF, Mehta AK, Tomashek KM, Wolfe CR, Ghazaryan V, Marconi VC, Ruiz-Palacios GM, Hsieh L, Kline S, Tapson V, Iovine NM, Jain MK, Sweeney DA, El Sahly HM, Branche AR, Regalado Pineda J, Lye DC, Sandkovsky U et al (2021) Baricitinib plus remdesivir for hospitalized adults with COVID-19. N Engl J Med 384:795-807. https://doi.org/10.1056/ NEJMoa2031994

11. Cao Y, Wei J, Zou L, Jiang T, Wang G, Chen L, Huang L, Meng F, Huang L, Wang N, Zhou X, Luo H, Mao Z, Chen X, Xie J, Liu J, Cheng H, Zhao J, Huang G et al (2020) Ruxolitinib in treatment of severe coronavirus disease 2019 (COVID-19): a multicenter, single-blind, randomized controlled trial. J Allergy Clin Immunol 146: 137-146.e3. https://doi.org/10.1016/j.jaci.2020.05.019

12. Guimarães PO, Quirk D, Furtado RH, Maia LN, Saraiva JF, Antunes MO, Kalil Filho R, Junior VM, Soeiro AM, Tognon AP, Veiga VC, Martins PA, Moia DDF, Sampaio BS, Assis SRL, Soares RVP, Piano LPA, Castilho K, Momesso RGRAP et al (2021) Tofacitinib in patients hospitalized with COVID-19 pneumonia. N Engl J Med 385:406-415. https://doi.org/10.1056/ NEJMoa2101643

13. Singh D, Bogus M, Moskalenko V, Lord R, Moran EJ, Crater GD, Bourdet DL, Pfeifer ND, Woo J, Kaufman E, Lombardi DA, Weng EY, Nguyen T, Woodcock A, Haumann B, Saggar R (2021) A phase 2 multiple ascending dose study of the inhaled pan-JAK inhibitor nezulcitinib (TD-0903) in severe COVID-19 [published online ahead of print, $2021 \mathrm{Jul}$ 1]. Eur Respir J 2021:2100673. https://doi.org/10.1183/13993003.00673-2021.

14. Atemnkeng F, Alataby H, Demirjian J, Kenne FM, Nfonoyim J (2021) A novel report on the compassionate use of baricitinib in treating a pediatric patient with severe symptoms of COVID-19 infection. J Med Cases 12:94-98. https://doi.org/10.14740/ jmc3629

15. Jorgensen SCJ, Burry L, Tse CLY, Dresser LD (2020) Baricitinib: impact on COVID-19 coagulopathy? Clin Infect Dis 2020: ciaa1208. https://doi.org/10.1093/cid/ciaa1208

16. Innes AJ, Cook LB, Marks S, Bataillard E, Crossette-Thambiah C, Sivasubramaniam G, Apperley J, Milojkovic D (2020) Ruxolitinib for tocilizumab-refractory severe COVID-19 infection. Br J Haematol 190:e198-e200. https://doi.org/10.1111/bjh.16979

Publisher's note Springer Nature remains neutral with regard to jurisdictional claims in published maps and institutional affiliations.

\section{Affiliations}

\section{Dimitrios Patoulias $^{1}$ (D) $\cdot$ Michael Doumas $^{1,2} \cdot$ Christodoulos Papadopoulos $^{3} \cdot$ Asterios Karagiannis $^{1}$}

1 Second Propedeutic Department of Internal Medicine, Aristotle University of Thessaloniki, General Hospital "Hippokration", Konstantinoupoleos 49, 54642 Thessaloniki, Greece

2 Veterans Affairs Medical Center, George Washington University, Washington, DC, USA
3 Third Department of Cardiology, Aristotle University of Thessaloniki, General Hospital "Hippokration", Thessaloniki, Greece 